\title{
Das doppelte Demokratiedefizit europäischer Sicherheits- und Verteidigungspolitik: Paradox eines kontrafaktischen Legitimitäts- modells parlamentarischer Kontrolle?
}

\author{
Julian Böcker und Oliver Schwarz
}

Die Debatte um eine parlamentarische Kontrolle in der europäischen Außen-, Sicherheitsund Verteidigungspolitik hat in den vergangenen Jahren an politischer Brisanz gewonnen. ${ }^{1}$ Auch wenn die Entscheidung zur Entsendung von nationalen Streitkräften im Rahmen einer Mission der Europäischen Union (EU) einstimmig im Rat entschieden werden muss, so bemängeln Kritiker eine unzureichende Beteiligung der nationalen Parlamente im Entscheidungsprozess der Gemeinsamen Sicherheits- und Verteidigungspolitik (GSVP). Zwar sind die Regierungen der 27 EU-Mitgliedstaaten gegenüber ihren nationalen Parlamenten direkt rechenschaftspflichtig, deren konkrete Kompetenzen weichen zum Teil jedoch stark voneinander $\mathrm{ab}$. Während es in manchen Mitgliedsländern einen Parlamentsvorbehalt zur Entsendung nationaler Streitkräfte gibt, werden andere Parlamente lediglich über die Entscheidungen ihrer Regierungen im Nachhinein informiert. Gemeinsam mit der konsultativen Rolle des Europäischen Parlaments (EP), das militärische GSVP-Missionen zwar intensiv diskutiert, aber keinen rechtlichen Einfluss auf die Entscheidung der Regierungen im Rat hat, führt dies einige Beobachter zu dem Schluss, dass die GSVP unter einem so genannten „doppelten Demokratiedefizit“2 leidet.

Dieser These liegt ein spezifisches normatives Legitimitätsmodell zugrunde, demzufolge die demokratische Legitimität der GSVP nur durch umfassende parlamentarische Kontrolle gewährleistet werden kann. Der konstitutive Parlamentsvorbehalt wird dabei als stärkster Garant demokratischer Legitimität identifiziert, da das Parlament in einer parlamentarischen Demokratie die einzige direkt vom Volk gewählte Institution darstellt. Bereits Giandomenico Majone hat jedoch in einem viel beachteten Beitrag daran erinnert, dass die Diskussion um das Demokratiedefizit der EU im Wesentlichen eine Frage der zugrunde liegenden Bewertungsstandards sei. ${ }^{3}$ Folglich falle der Befund eines demokratischen Defizits in der Regel umso deutlicher aus, je höher die Demokratiestandards gesetzt werden. Gerade weil bei der EU-Legitimitätsdebatte ${ }^{4}$ sehr umstritten ist, welches Demokratieverständnis für das Regie-

1 Vgl. Julian Böcker, Demokratiedefizit der Sicherheits- und Verteidigungspolitik der EU? Analyse des deutschen, britischen und Europäischen Parlaments, Baden-Baden 2012; Giovanna Bono, Challenges of Democratic Oversight of EU Security Policies, in: European Security, 15. Jg. (2006), H. 4, S. 431 - 449; Sandra Dieterich / Hartwig Hummel / Stefan Marschall, Strengthening Parliamentary "War Powers" in Europe: Lessons from 25 National Parliaments (Policy Paper No. 27), Genf 2009; René Lüddecke, Parlamentarisierung der nationalen Außenpolitik, Baden-Baden 2010; Jürgen Mittag, The Parliamentary Dimension of CFSP/ESDP. Options for the European Convention (Study submitted to the European Parliament), Brüssel 2003; Wolfgang Wagner, The Democratic Legitimacy of European Security and Defence Policy (Occasional Paper No. 57), Paris 2005.

2 Hans Born / Heiner Hänggi, The 'Double Democratic Deficit'. Parliamentary Accountability and the Use of Force Under International Auspices, Aldershot 2004.

3 Vgl. Giandomenico Majone, Europe's 'Democratic Deficit': The Question of Standards, in: European Law Journal, 4. Jg. (1998) H. 1, S. 5 - 28.

4 Vgl. Andreas Follesdal / Simon Hix, Why There is a Democratic Deficit in the EU: A Response to Majone and Moravcsik, in: Journal of Common Market Studies, 44. Jg. (2006), H. 3, S. 533 - 
ren im europäischen Mehrebenensystem als angemessen erscheint, sollte der Idealtypus des verfassungsrechtlich verankerten parlamentarischen Vorbehalts als kontrafaktisches Legitimitätsmodell hinterfragt und an der realen Praxis überprüft werden. ${ }^{5}$

Der vorliegende Aufsatz hat daher zum Ziel, die unterschiedlichen parlamentarischen Kontrollregime innerhalb der EU aufzuzeigen und basierend auf einer empirischen Analyse eine belastbare Antwort auf die Frage zu finden, inwieweit die GSVP tatsächlich unter einem Demokratiedefizit leidet. Hierzu wird zunächst in einem ersten Schritt ein vergleichender Überblick über die Beteiligung der 27 nationalen Parlamente im Entscheidungsprozess der GSVP gegeben. Mithilfe der hieraus resultierenden Kategorisierung wird das normative Legitimitätsmodell des konstitutiven Parlamentsvorbehalts im europäischen Kontext verortet und ausführlich am Beispiel des Deutschen Bundestages vorgestellt. Dem werden die Kontroll- und Mitwirkungsrechte des britischen Unterhauses gegenübergestellt. Ein Überblick über die Beteiligungsrechte des EP schließt die exemplarische Darstellung ab, in die Einschätzungen, Meinungen und Wahrnehmungen von Abgeordneten des deutschen und britischen Parlaments sowie des Europäischen Parlaments eingebettet werden, die durch qualitative Experteninterviews gewonnen wurden. ${ }^{6}$

\section{Demokratisches Defizit durch mangelnde parlamentarische Kontrolle?}

Seit Max Webers Ausführungen zur „legitimen Herrschaft“7 zählt die Frage, mit welchen Gründen sich autoritativ herbeigeführte Entscheidungen rechtfertigen lassen, zu den Kernbeständen der sozialwissenschaftlichen Forschung. Obwohl zu Beginn der 1990er Jahre zunächst das „Ende der Legitimitätstheorien“8 konstatiert wurde, erlebt die theoretische Auseinandersetzung mit dem Legitimitätsbegriff seither eine Art Blütezeit. Dazu hat im Wesentlichen die Diskussion über das „Demokratiedefizit“ der EU beigetragen. ${ }^{9}$ Joseph Weiler, Ulrich Haltern und Franz Mayer verdichteten die wesentlichen Argumentationsstränge dieser Auseinandersetzung in einer so genannten Standardversion ${ }^{10}$ des europäischen Demokratiedefizits. Die darin skizzierten Argumente bestimmen im Großen und Ganzen

562; Andrew Moravcsik, In Defence of the 'Democratic Deficit': Reassessing Legitimacy in the European Union, in: Journal of Common Market Studies, 40. Jg. (2002), H. 4, S. $603-624$.

5 Vgl. Andreas Wimmel, Demokratische Legitimität europäischen Regierens: ein Labyrinth ohne Ausgang?, in: Integration, 31. Jg. (2008), H. 1, S. 48 - 64, S. 60.

6 Insgesamt wurden 34 qualitative Experteninterviews in der Zeit von Mai bis Juli 2009 geführt, wobei mindestens zehn in jedem der ausgewählten Parlamente stattgefunden haben. Der Volltext der transkribierten Interviews befindet sich online unter: http://www.nomos-shop.de/14338 (Abruf am 6. Oktober 2012).

7 Max Weber, Staatssoziologie. Soziologie der rationalen Staatsanstalt und der modernen politischen Parteien und Parlamente, Berlin 1966, S. 107.

8 Klaus von Beyme, Theorie der Politik im 20. Jahrhundert. Von der Moderne zur Postmoderne, Frankfurt am Main 1992, S. 199.

9 Vgl. Peter Graf Kielmansegg, Integration und Demokratie, in: Peter Jachtenfuchs / Beate Kohler-Koch, Europäische Integration, Opladen 1996, S. 47 - 71; Frank Schimmelfennig, Legitimate Rule in the European Union. The Academic Debate (Tübinger Arbeitspapier zur Internationalen Politik und Friedensforschung 27), Tübingen 1996; Michael Zürn, Über den Staat und die Demokratie im europäischen Mehrebenensystem, in: PVS, 37. Jg. (1996), H. 1, S. 27 - 55.

10 Vgl. Joseph. H. H. Weiler / Ulrich R. Haltern / Franz Mayer, European Democracy and its Critique, in: West European Politics, 18. Jg. (1995) H. 3, S. $4-39$, S. 6. 
auch heute noch die Diskussion, so dass Andreas Follesdal und Simon Hix unlängst eine „aktualisierte Standardversion“11 des europäischen Demokratiedefizits präsentiert haben. In beiden Versionen findet sich dabei der Vorwurf der Entparlamentarisierung wieder.

Diese ernüchternde Einschätzung speist sich vor allem aus zwei ideengeschichtlichen Hauptlinien, die das traditionelle Paradigma parlamentarischer Rechtsetzung begründet haben. Hierbei handelt es sich zum einen um die Volkssouveränitätslehre Jean-Jacques Rousseaus und zum anderen um die Genese der parlamentarischen Monarchie im Vereinigten Königreich. Während die von Rousseau formulierte Volkssouveränität durch das ganze Volk lediglich ein Ideal blieb, bildete sich im britischen System das Grundprinzip der Parlamentssouveränität praktisch heraus. Speziell im deutschen Diskurs spiegelt sich diese Sichtweise unverändert in einem vorwiegend inputorientierten Demokratieverständnis wider, dass die Legitimität politischer Entscheidungen primär durch die Zustimmung der Beherrschten und die möglichst authentische Repräsentation der Mitglieder einer Gemeinschaft gewährleistet sieht. ${ }^{12}$ Klagen über den Niedergang der Parlamente sind somit „so alt wie der Parlamentarismus selbst"13. Im Zuge der europäischen Integration haben Vorwürfe dieser Art jedoch erheblich zugenommen. Es findet sich kaum eine einschlägige Publikation, in der die Europäisierung der nationalen Regierungssysteme nicht als schleichender Prozess eines relativen Machtverlustes der Parlamente kritisiert wird. ${ }^{14}$

Von dem Vorwurf einer schleichenden Entparlamentarisierung oder zumindest einer unverhältnismäßigen Exekutivlastigkeit politischer Entscheidungen bleibt naturgemäß auch die intergouvernementale Gemeinsame Außen- und Sicherheitspolitik (GASP) nicht verschont. Trotz der jüngsten Änderungen durch den Vertrag von Lissabon handelt es sich bei der EU unverändert um einen „zusammengesetzten “ 15 und deshalb in vielerlei Hinsicht heterogenen Akteur. Da die Außenbeziehungen eines Nationalstaats traditionell ein Vorrecht der Exekutive darstellen, sind die Einflussmöglichkeiten der Parlamente hier generell weniger stark ausgeprägt. Klaus von Beyme erklärte den Bereich der Außenpolitik sogar zum "Sonderfall der Ohnmacht des Parlaments" ${ }^{6}$. Dies trifft im Besonderen auf die GSVP zu, bei der die EU-Mitgliedstaaten ihre jeweiligen Prärogativen bewahren und nur allzu oft nationalstaatliche Positionen die Agenda bestimmen. ${ }^{17}$ Die Folge ist ein komplexes Governance-System der außenpolitischen Konfliktbearbeitung in der EU, in dem die Parlamente

11 Andreas Follesdal/ Simon Hix, a.a.O. (Fn. 4), S. 534.

12 Vgl. Fritz W. Scharpf, Regieren in Europa. Effektiv und demokratisch?, Frankfurt am Main 1999, S. 16.

13 Klaus von Beyme, Niedergang der Parlamente. Internationale Politik und nationale Entscheidungshoheit, in: Internationale Politik, 53. Jg. (1998), H. 4, S. 20 - 30, S. 21.

14 Vgl. Tanja Börzel, Europäisierung und innerstaatlicher Wandel: Zentralisierung und Entparlamentarisierung, in: PVS, 41. Jg. (2000), H. 2, S. 225 - 250; Andreas Follesdal/ Simon Hix, a.a.O. (Fn. 4); Klaus Goetz, Europäisierung der öffentlichen Verwaltung - oder europäische Verwaltung?, in: Jörg Bogumil / Werner Jann / Frank Nullmeier, Politik und Verwaltung. Auf dem Weg zu einer postmanagerialen Verwaltungsforschung (PVS-Sonderheft 37), Wiesbaden 2006, S. 472 - 490; Andrew Moravcsik, Why the European Community Strengthens the State: Domestic Politics and International Cooperation (Working Paper No. 52), Cambridge 1994.

15 Hans-Georg Ehrhart, Die Sicherheitspolitik der EU im Werden, in: Internationale Politik, 59. Jg. (2004), H. 4, S. 33 - 41, S. 34.

16 Klaus von Beyme, a.a.O. (Fn. 13), S. 22.

17 Vgl. Selma Aposkitis, Europäisierung oder Renationalisierung. Nationalstaatliche Positionen in der Europäischen Sicherheits- und Verteidigungspolitik (ESVP), Baden-Baden 2006. 
eine untergeordnete Rolle spielen. Nicht zuletzt deshalb kommt es in der Literatur daher oft zum Vorwurf eines „doppelten Demokratiedefizits“ in diesem Politikbereich.

\subsection{Die Diskussion um das doppelte Demokratiedefizit in der GSVP}

Das Konzept des „doppelten Demokratiedefizits“ bezieht sich primär auf die Wechselwirkung zwischen einem EP, das unzureichend mit formalen Einflüssen auf die Entscheidungsfindung der GSVP ausgestattet ist, und den zwar formal einflussreicheren, aber in der Praxis oft schlechter informierten nationalen Parlamenten. Auf nationaler Ebene gebe es zudem aufgrund von unterschiedlichen demokratischen Traditionen keine minimalen Standards parlamentarischer Kontrolle, was zu einem „kumulativen demokratischen Defizit“18 führe. Als stärkstes Instrument parlamentarischer Kontrolle gilt das konstitutionelle Recht eines Parlaments, über den Einsatz von Soldaten bestimmen zu können, das heißt die in der Verfassung verankerte Verpflichtung der Regierung, das Parlament im Vorfeld oder nach einer Entscheidung zum Entsenden von Soldaten um eine Autorisierung zu bitten. ${ }^{19}$ Ein solches parlamentarisches Recht kann sowohl bindenden als auch nicht bindenden Charakter haben. Aufgrund der unterschiedlichen Demokratiemodelle in der EU ist ein derartiger Parlamentsvorbehalt aber nur in einigen Mitgliedstaaten vorhanden. Diese ungleichmäßigen parlamentarischen Kontrollmöglichkeiten werden häufig als Argument für ein Demokratiedefizit in der GSVP herangezogen. ${ }^{20}$

Auf der internationalen Ebene sei die parlamentarische Kontrolle gar noch weniger verbreitet, wenn es um die Anwendung von Gewalt seitens internationaler Organisationen und ad hoc-Koalitionen geht. Dies liege primär an der ausschließlich intergouvernementalen Struktur internationaler Organisationen. Die EU sei hier zwar durch ihr direkt gewähltes Parlament die Ausnahme, allerdings könne das EP trotz erheblicher Ressourcen und eines starken politischen Willens seinen Einfluss nur bedingt geltend machen. ${ }^{21}$ Da es sich bei der GSVP um einen intergouvernementalen Politikbereich handelt, sollten es primär die nationalen Parlamente sein, die die parlamentarische Kontrolle durchführen. Aber selbst wenn diese mit ausreichend formalen Befugnissen ausgestattet wären, fehlten ihnen oft die nötigen Mechanismen, um rechtzeitig Informationen, insbesondere in Bezug auf die Positionen der anderen EU-Mitgliedstaaten, zu erhalten. Das EP habe hier zwar einen klaren Standortvorteil und könne die Verantwortlichen der in Brüssel befindlichen EU-Institutionen direkt in die Ausschüsse zitieren, allerdings mangelt es ihm an formalen Befugnissen, um die demokratische Legitimität der GSVP zu gewährleisten. ${ }^{22}$

Verschiedene Studien haben jedoch inzwischen aufgezeigt, dass der pauschale Vorwurf einer unzureichenden parlamentarischen Kontrolle der GSVP nicht per se haltbar ist. ${ }^{23}$ Dies

18 Hans Born / Heiner Hänggi, The Use of Force under International Auspices: Strengthening Parliamentary Accountability (Policy Paper No. 7), Genf 2005, S. 21.

19 Vgl. ebenda, S. 5.

20 Vgl. Giovanna Bono, a.a.O. (Fn. 1), S. 440; Hans Born / Heiner Hänggi, a.a.O. (Fn. 2), S. 12.

21 Vgl. Hans Born / Heiner Hänggi, a.a.O. (Fn. 18), S. 5.

22 Vgl. Ester Barbé, The Evolution and Democratic Accountability of CFSP Institutions, in: International Spectator, 39. Jg. (2004), H. 2, S. 47 - 60, S. 53.

23 Vgl. Julian Böcker, a.a.O. (Fn. 1); Hans Born / Alex Dowling / Teodora Fuior / Suzana Anghel, Parliamentary Oversight of ESDP Missions (Policy Paper No. 28), Genf 2008; Andrew Moravcsik, 
liegt unter anderem an der sich wandelnden Rolle des Europäischen Parlaments. ${ }^{24}$ Gerade im Vergleich mit Entscheidungsprozessen zu Operationen der Vereinten Nationen bieten sich den Parlamenten innerhalb der EU deutlich mehr Beteiligungsmöglichkeiten. ${ }^{25} \mathrm{Im}$ Folgenden soll daher zunächst ein vergleichender Überblick über die Beteiligung nationaler Parlamente am Entscheidungsprozess der GSVP gegeben werden, bevor drei ausgesuchte parlamentarische Kontrollregime exemplarisch näher untersucht werden.

\subsection{Eine Kategorisierung parlamentarischer Kontrollregime in der EU}

In der Literatur gibt es eine Reihe von Kategorisierungen, die zwischen unterschiedlichen Typen parlamentarischer Kontrolle unterscheiden. ${ }^{26}$ Da grundsätzlich jedes Parlament die Sicherheits- und Verteidigungspolitik zumindest debattieren kann, sollte der Fokus einer Kategorisierung insbesondere auf der legislativen Einflussnahme auf dem Entscheidungsprozess der GSVP liegen: Hat das Parlament die Möglichkeit, den Einsatz von Soldaten zu verhindern beziehungsweise ist seine Zustimmung grundsätzlich erforderlich? Ein wichtiges Merkmal in diesem Zusammenhang ist die Unterscheidung zwischen ex ante- und ex postKontrolle.

Die ex ante-Kontrolle bezieht sich auf jegliche Form parlamentarischer Kontrolle, die im Vorfeld des Entsendens von Soldaten stattfindet, unabhängig davon, ob eine Gemeinsame Aktion im Rat verabschiedet wurde oder nicht. Das wichtigste Instrument dabei ist das Recht des Parlaments, den Vorschlag der Regierung, Truppen zu entsenden, annehmen, ändern oder ablehnen zu können. Zur parlamentarischen ex ante-Kontrolle gehört aber auch der parlamentarische Einfluss innerhalb des Entscheidungsprozesses durch die Ausübung von Haushaltskontrollrechten sowie durch öffentliche Anhörungen und Fragestunden mit Experten und Regierungsvertretern im Vorfeld einer GSVP-Mission. Die ex post-Kontrolle hingegen bezieht sich auf jede Form parlamentarischer Kontrolle, die stattfindet, nachdem Soldaten bereits in die Einsatzgebiete geschickt worden sind. Dazu zählen sowohl die Möglichkeit, Berichte und Studien zu erstellen, das Einsatzmandat zu verlängern oder den Abzug der Soldaten zu fordern, Fragestunden und das Zitieren von Ministern sowie Regierungsbeamten vor einzelne Ausschüsse beziehungsweise das Plenum sowie Delegationsreisen in die Einsatzgebiete.

a.a.O. (Fn. 4); Daniel Thym, Parliamentary Involvement in European International Relations, in: Marise Cremona / Bruno de Witte, EU Foreign Relations Law - Constitutional Fundamentals, Oxford 2008, S. $201-232$.

24 Vgl. Elisabeth Wisniewski, Coming to Terms with the 'Legitimacy Crisis' of European Foreign Politics (RECON Online Working Paper 28), Oslo 2011.

25 Vgl. Nicolai von Ondarza, Legitimatoren ohne Einfluss? Nationale Parlamente in Entscheidungsprozessen zu militärischen EU- und VN-Operationen im Vergleich, Baden-Baden 2012, S. 21.

26 Vgl. Hans Born / Alex Dowling / Teodora Fuior / Suzana Anghel, a.a.O. (Fn. 23); Hans Born / Heiner Hänggi, a.a.O. (Fn. 18); Sandra Dieterich / Hartwig Hummel / Stefan Marschall, a.a.O. (Fn. 1); Jaime Mayor Oreja, Parliamentary Oversight of the European Security and Defence Policy, in: Karl von Wogau, The Path to European Defence. New Roads, New Horizons, London 2009; Mafalda Troncho, National Parliamentary Scrutiny of Intervention Abroad by Armed Forces Engaged in International Missions: The Current Position in Law (Document A/1762), Brüssel 2001; Wolfgang Wagner, The Democratic Control of Military Power Europe, in: Journal of European Public Policy, 13. Jg. (2006), H. 2, S. $200-216$. 
Dieser zeitlichen Logik folgend wird zwischen vier Modellen parlamentarischer Kontrolle im Rahmen der GSVP unterschieden. ${ }^{27}$ Zur ersten Gruppe gehören die Parlamente, die einen Einsatz autorisieren, noch bevor die Gemeinsame Aktion im Rat angenommen worden ist. Die Parlamente haben somit einen erheblichen und frühzeitigen Einfluss auf die Positionierung ihrer Regierung im Rat. Zur zweiten Gruppe zählen die Parlamente, die eine Mission genehmigen müssen, nachdem die Gemeinsame Aktion angenommen worden ist, aber noch bevor die Soldaten in Krisengebiete entsandt werden. Im dritten Modell haben die Parlamente lediglich eine konsultative Stellung im Entscheidungsprozess inne. Auch wenn sie die Missionen im Rahmen der GSVP diskutieren, teilweise sogar im Vorfeld der Annahme einer Gemeinsamen Aktion, ist die Regierung nicht auf die Zustimmung des Parlaments angewiesen und kann selbst im Falle einer Ablehnung die Gemeinsame Aktion unterstützen und Soldaten entsenden. Zur letzten Gruppe gehören die Länder, in denen die Parlamente keine substanzielle Rolle im Rahmen der GSVP spielen. Sie werden lediglich über GSVP-Missionen informiert, selbst wenn die Regierung Soldaten zur Verfügung stellt.

Auf Basis dieser Kategorisierung und nach einer ergänzenden Literaturauswertung ergibt sich folgende Klassifizierung von 26 nationalen Parlamenten in der EU. ${ }^{28}$

\begin{tabular}{|c|c|c|}
\hline & & EU-Mitgliedsstaaten* \\
\hline \multirow{4}{*}{$\begin{array}{l}\text { Einfluss des } \\
\text { Parlaments }\end{array}$} & $\begin{array}{l}\text { Autorisierung vor } \\
\text { Gemeinsamer Aktion }\end{array}$ & Finnland, Luxemburg, Schweden \\
\hline & $\begin{array}{l}\text { Autorisierung vor } \\
\text { Entsendung }\end{array}$ & $\begin{array}{l}\text { Bulgarien, Deutschland, Estland, Irland, Italien, Lettland, } \\
\text { Litauen, Niederlande, Österreich, Slowakei, Slowenien, } \\
\text { Spanien, Tschechien, Zypern }\end{array}$ \\
\hline & $\begin{array}{l}\text { Konsultation } \\
\text { des Parlaments }\end{array}$ & $\begin{array}{l}\text { Belgien, Frankreich, Großbritannien, Malta, Polen, Portugal, } \\
\text { Ungarn }\end{array}$ \\
\hline & $\begin{array}{l}\text { Information } \\
\text { des Parlaments }\end{array}$ & Griechenland, Rumänien \\
\hline
\end{tabular}

Die Beteiligung der nationalen Parlamente am Entscheidungsprozess der GSVP fällt offenkundig sehr unterschiedlich aus. Während manche wesentlichen Einfluss auf das Entsenden von Soldaten haben, teilweise sogar im Vorfeld einer Entscheidung im Rat, werden andere lediglich konsultiert. Einige Parlamente haben keinen Einfluss auf den Entscheidungsprozess, weder vor noch nach einer Gemeinsamen Aktion und werden nur über die Entscheidungen der Regierungschefs informiert (vgl. Tabelle 1).

In der ersten Gruppe finden sich lediglich drei EU-Mitgliedstaaten. Allein Finnland, Luxemburg und Schweden beraten in ihren Parlamenten alle anstehenden GSVP-Missionen noch bevor die Regierungsvertreter sich in den Rat begeben und eine Gemeinsame Aktion beschließen. In Schweden ist dieser Grundsatz sogar in der Verfassung verankert. Nach Kapitel 10 Paragraph 9 dürfen die schwedischen Streitkräfte nur dann zum Einsatz kommen,

27 Vgl. Hans Born / Alex Dowling / Teodora Fuior / Suzana Gavrilescu, Parliamentary Oversight of Civilian and Military ESDP Missions: The European and National Levels, Brüssel 2007; Hans Born / Alex Dowling / Teodora Fuior / Suzana Anghel, a.a.O. (Fn. 23).

28 Vgl. Julian Böcker, a.a.O. (Fn. 1), S. 143 ff. 
wenn der Reichstag dies explizit genehmigt. ${ }^{29}$ Auch wenn es in speziellen Gesetzen noch einige Ausnahmen zu dieser Regel gibt, so hat die politische Praxis gezeigt, dass die schwedische Regierung grundsätzlich im Vorfeld einer Entscheidung auf europäischer Ebene die Genehmigung des Parlaments einholt. Auch die Verfassung Luxemburgs sieht ein derartiges Prozedere vor. Nach Artikel 37 ist der Großherzog der Befehlshaber aller Streitkräfte, die nur zum Einsatz gelangen können, wenn er hierzu von der Abgeordnetenkammer ermächtigt wird. Nach einem speziellen Gesetz aus dem Jahr 1992 für internationale Friedensunterstützungsmissionen entscheidet die Regierung nach einer Konsultation der zuständigen Gremien des Parlaments. Auch wenn die Verfassung damit nicht ganz im Einklang mit dem Gesetz von 1992 steht, so hat die Praxis gezeigt, dass Luxemburg zu den Ländern gehört, die eine Autorisierung ihres Parlaments einholen, noch bevor eine Gemeinsame Aktion beschlossen wird. ${ }^{30}$ In Finnland wurde die parlamentarische Kontrolle zuletzt 2006 durch den „Act on Military Crisis Management" (inoffizielle Übersetzung des Verteidigungsministeriums) geregelt. Gemäß Paragraph 3 des Dokuments muss die finnische Regierung den Auswärtigen Ausschuss des Parlaments konsultieren, noch bevor sie einen Vorschlag zum Entsenden von Soldaten einreicht. Im Falle einer anspruchsvollen militärischen Herausforderung im Rahmen einer Krisenmanagement-Operation oder im Falle einer Operation ohne ein Mandat des UN-Sicherheitsrates muss die Regierung das Parlament konsultieren und ihm einen entsprechenden Bericht vorlegen. Nach der Zustimmung des Parlaments macht der Minister für Auswärtige Angelegenheiten einen offiziellen Vorschlag an den Präsidenten zum Entsenden der Soldaten. ${ }^{31}$

Nur in zwei EU-Mitgliedstaaten haben die Parlamente nahezu keinen Einfluss auf die GSVP. Die Beteiligung des griechischen Parlaments an Entscheidungen zum Entsenden von Soldaten ist marginal bis praktisch gar nicht vorhanden. Der Verteidigungsminister informiert lediglich den Ausschuss für Verteidigung und Auswärtige Angelegenheiten des Parlaments. ${ }^{32}$ Auch in Rumänien ist das Parlament weder in zivile noch in militärische GSVPMissionen involviert. Die Entscheidung, Soldaten auf internationale Missionen im Rahmen der GSVP zu entsenden, liegt allein beim Präsidenten, der das Parlament innerhalb von fünf Tagen informieren muss. Ein zusätzliches Gesetz zur Zusammenarbeit zwischen dem Parlament und der Regierung in europäischen Angelegenheiten wurde inzwischen zwar auf den Weg gebracht ${ }^{33}$, der parlamentarische Ratifikationsprozess ist jedoch noch nicht abgeschlossen.

In insgesamt 14 Staaten der EU benötigt die Entsendung nationaler Streitkräfte grundsätzlich eine parlamentarische Zustimmung. In sieben weiteren Ländern bedarf es hingegen lediglich einer Konsultation des nationalen Parlaments. Da es sich bei diesen beiden Gruppen um die größten handelt, wird im Folgenden aus jeder jeweils ein Staat exemplarisch vorgestellt. Dafür bieten sich Großbritannien und Deutschland aus mehreren Gründen an. Zum einen zählen sie zu den größten und einflussreichsten Mitgliedstaaten der EU und können damit die weitere Entwicklung der GSVP wesentlich vorantreiben. Zum anderen

29 Vgl. Wolfgang Wagner, a.a.O. (Fn. 26), S. 53 f.

30 Vgl. Sandra Dieterich / Hartwig Hummel / Stefan Marschall, a.a.O. (Fn. 1), S. 12.

31 Vgl. Hans Born / Alex Dowling / Teodora Fuior / Suzana Anghel, a.a.O. (Fn. 23), S. 32 ff.

32 Vgl. Wolfgang Wagner, a.a.O. (Fn. 26), S. 43.

33 Vgl. COSAC, Tenth Bi-annual Report: Developments in European Union, Procedures and Practices Relevant to Parliamentary Scrutiny, Paris 2008, S. 28. 
haben sie sehr unterschiedliche Leitbilder für eine vertiefte Sicherheits- und Verteidigungspolitik auf europäischer Ebene. Während Deutschland eine Vertiefung der GASP/GSVP ausdrücklich begrüßt und die langfristige Entwicklung einer parlamentarisch kontrollierten europäischen Armee anstrebt, so lehnt Großbritannien jede weitere Integration in diesem Politikbereich ab und setzt neben einer verstärkten Anbindung an die (NATO) auf die Beibehaltung der intergouvernementalen Struktur der GSVP. ${ }^{34}$ Darüber hinaus ist die Rolle des deutschen und britischen Parlaments im Entscheidungsprozess ebenfalls sehr unterschiedlich ausgeprägt. Nach dem Urteil des Bundesverfassungsgerichts (BVerfG) aus dem Jahr 1994 und dem Parlamentsbeteiligungsgesetz aus dem Jahr 2005 muss jede Entsendung von deutschen Soldaten im Vorfeld vom Bundestag genehmigt werden. Diese Regelung gilt jedoch nicht für den Einsatz von Polizisten im Rahmen von zivilen GSVP-Missionen. In Großbritannien liegt das Hoheitsrecht zum Entsenden von Soldaten traditionell beim König beziehungsweise beim Premierminister. Eine Autorisierung des Parlaments ist nicht erforderlich. Das britische Unterhaus hat keinen rechtlichen Einfluss auf die Entscheidung des Premierministers zur Entsendung von Soldaten. Allerdings debattiert das House of Commons, ebenso wie das House of Lords, GSVP-Missionen vor und insbesondere nach dem Entsenden von Soldaten in Krisenregionen.

\section{Parlamentarische Kontrolle in der GSVP: Unterschiede, Gemeinsamkeiten und Perspektiven}

Die große Bandbreite parlamentarischer Kontrollrechte im Bereich der Sicherheits- und Verteidigungspolitik spiegelt die kulturelle Vielfalt Europas und die historisch gewachsenen Demokratieverständnisse und Legitimitätsvorstellungen wider. Das normative Legitimitätsmodell des konstitutiven Parlamentsvorbehalts kann daher nicht ohne eine genaue Prüfung als Best-Practice-Beispiel herangezogen werden. Im Folgenden soll, dieses normative Legitimitätsmodell im europäischen Kontext verortet und erörtert werden, ob eine Anpassung parlamentarischer Kontrollrechte im Hinblick auf die Gewährleistung der demokratischen Legitimität der GSVP notwendig und überhaupt wünschenswert ist.

\subsection{Deutschland: Das Musterbeispiel?}

Der konstitutive Parlamentsvorbehalt des Bundestages ist der historischen Entwicklung der Bundesrepublik Deutschland im 20. Jahrhundert geschuldet und ist der zentrale Bestandteil des deutschen Leitbilds parlamentarischer Außenpolitik. ${ }^{35}$ Nach dem Ende des Zweiten Weltkriegs bildeten die ablehnende Haltung gegenüber der Anwendung militärischer Gewalt und ein Bekenntnis zum Multilateralismus die wesentlichen Pfeiler deutscher Sicherheitspolitik. Das Grundgesetz, das bei seiner Annahme im Jahr 1949 keine Erwähnung von

34 Vgl. Julian Böcker, a.a.O. (Fn. 1), S. 152 ff.

35 Vgl. Rafael Biermann, Der Deutsche Bundestag und die Auslandseinsätze der Bundeswehr. Zur Gratwanderung zwischen exekutiver Prärogative und legislativer Mitwirkung, in: ZParl, 35. Jg. (2004), H. 4, S. 607 - 626; Dieter Wiefelspütz, Der konstitutive wehrverfassungsrechtliche Parlamentsbeschluss, in: ZParl, 38. Jg. (2007), H. 1, S. 3 - 16; ders., Der Einsatz bewaffneter deutscher Streitkräfte und der konstitutive Parlamentsvorbehalt, Baden-Baden 2003. 
Streitkräften beinhaltete, wurde erst auf Drängen der Alliierten 1969 so erweitert, dass eine gesetzliche Grundlage für den Einsatz der Bundeswehr im Verteidigungsfalle entstand. ${ }^{36}$ Bis zum Ende des Kalten Krieges hatte sich in der Bundesrepublik eine „Kultur der Zurückhaltung "37 ausgeprägt, die jeglichen Einsatz der Bundeswehr mit Ausnahme des Verteidigungsfalles ablehnte. Mit dem Zusammenbruch der Sowjetunion, den daraus resultierenden neuen geopolitischen Herausforderungen und der deutschen Wiedervereinigung wuchsen jedoch die allgemeinen Erwartungen an eine neu ausgerichtete deutsche Außenpolitik. ${ }^{38}$ Die Entscheidung der Bundesregierung, deutsche Soldaten nicht aus der NATOFlotte abzuziehen, die im Rahmen eines UN-Sicherheitsmandats die Flugverbotszone über Bosnien 1993 überwachen sollte, wurde so heftig kritisiert, dass die Opposition eine Klage beim Bundesverfassungsgericht einreichte. Das Gericht stellte sich jedoch hinter die Entscheidung der Bundesregierung und hob als begründende Faktoren insbesondere die „Bündnissolidarität“ und die „Verlässlichkeit als Bündnispartner“39 hervor.

Das Urteil vom 12. Juli 1994 gilt als Meilenstein in der verfassungsrechtlichen Klärung des Einsatzes von Streitkräften im Ausland. Das Bundesverfassungsgericht unterstrich darin nicht nur die generelle Möglichkeit von Out-of-area-Einsätzen für die Bundeswehr, sondern schrieb darüber hinaus vor, dass vor jedem einzelnen Einsatz die vorherige Zustimmung des Bundestages einzuholen sei. Die Bundeswehr sei „nicht als Machtpotenzial allein der Exekutive zu überlassen, sondern als, Parlamentsheer' in die demokratisch rechtsstaatliche Verfassungsordnung einzufügen “ 40 . Das Urteil des Bundesverfassungsgerichts ist damit in zweierlei Hinsicht von großer Bedeutung: Zum einen hat es Deutschlands Rolle im internationalen System gestärkt, da die Bundesrepublik in ihrem neu verfolgten Kurs - entgegen der NichtIntervention - bekräftigt wurde. Zum anderen entstand damit die Parlamentsarmee, die dem Bundestag eine völlig neue Rolle in der Außenpolitik einbringen sollte.

Diese einflussreiche Rolle wurde mit dem Parlamentsbeteiligungsgesetz, das die Beteiligung des Bundestages an Einsatzentscheidungen bewaffneter Streitkräfte im Ausland regelt, im Jahr 2005 institutionalisiert. ${ }^{41}$ Damit ist nicht nur die vorherige Zustimmung des Bundestages notwendig. Die Bundesregierung ist ferner verpflichtet, das Parlament „regelmäßig“ über den Verlauf der Einsätze und über die Entwicklung im Einsatzgebiet zu unterrichten (\$ 6 ParlBG). Diese Berichte enthalten Informationen über die politische Situation im Einsatzgebiet, die allgemeine Sicherheit und sicherheitspolitische Vorfälle sowie zu Truppenbesuchen und zur allgemeinen Lage der Soldaten vor Ort. ${ }^{42}$ Zusätzlich hat der Bundestag Einflussmöglichkeiten bei der parlamentarischen Kontrolle von militärischen GSVP-Mis-

36 Vgl. Jörn Thießen / Ulrich Plate, Bundeswehr und Parlament, in: Hans J. Gießmann / Armin Wagner (Hrsg.), Armee im Einsatz. Grundlagen, Strategien und Ergebnisse einer Beteiligung der Bundeswehr, Baden-Baden 2009, S. 148 - 163, S. 148.

37 Wolfgang Wagner, Für Europa sterben? Die demokratische Legitimation der Europäischen Sicherheits- und Verteidigungspolitik. Hessische Stiftung für Friedens- und Konfliktforschung (HSFKReport 3), Frankfurt am Main 2004, S. 1 - 29, S. 18.

38 Vgl. Jürgen Mittag, a.a.O. (Fn. 1), S. 164.

39 Wolfgang Wagner, a.a.O. (Fn. 37), S. 19.

40 BVerfGE 90, S. 286, S. 382.

41 Vgl. Phillip Scherrer, Das Parlament und sein Heer. Das Beteiligungsgesetz, Berlin 2010; Dieter Wiefelspütz, Das Parlamentsheer, Berlin 2005; ders., Der Auslandseinsatz der Bundeswehr und das Parlamentsbeteiligungsgesetz, Frankfurt am Main 2008.

42 Vgl. Hans Born / Alex Dowling / Teodora Fuior / Suzana Gavrilescu, a.a.O. (Fn. 27), S. 35. 
sionen. Diese zeigen sich in erster Linie im Rahmen von Debatten im Verteidigungsausschuss (Informationsrecht), in Form von Besuchen von Abgeordneten in den Einsatzgebieten (Besuchsrecht) und bei der Haushaltskontrolle (Haushaltskontrollrecht). Im Gegensatz zu vielen anderen Ländern in Europa, wo die Parlamente den Haushalt für GSVP-Missionen lediglich im jährlichen Gesamthaushalt verabschieden, erhält der Bundestag nach dem Parlamentsbeteiligungsgesetz Auskunft über die bevorstehenden Kosten jedes einzelnen Streitkräfteeinsatzes und stimmt über diese im Vorfeld des Einsatzes ab (\$ 3 ParlBG). Der „power of the purse“ als parlamentarisches Kontrollinstrument kommt so in Deutschland eine ganz besondere Bedeutung zu. ${ }^{43}$

Insgesamt kann man zusammenfassen, dass zwar die Vorlage und konkrete Ausgestaltung eines Bundeswehreinsatzes in den Händen der Exekutive verbleibt, was auch vom BVerfG so bestätigt wurde. Dennoch hat der Bundestag eine Vielzahl an parlamentarischen ex anteund ex post-Kontrollinstrumenten, einschließlich eines Rückholrechts von Soldaten, um die Regierung effizient und zeitnah zu kontrollieren ( $\$ 8$ ParlBG). Somit gehört der Bundestag zu den Parlamenten in Europa, die aufgrund einer klaren gesetzlichen Grundlage und einer langjährigen parlamentarischen Praxis „zur obersten Kategorie parlamentarischer war powers, also zu den Staaten mit dem höchsten Grad an Demokratisierung im Bereich der Sicherheitspolitik“44 gezählt werden können.

In Deutschland ist die Beteiligung des Bundestages am Entscheidungsprozess für Auslandseinsätze der Bundeswehr somit inhärenter Bestandteil des Demokratieverständnisses. Das normative Legitimitätsmodell des konstitutiven Parlamentsvorbehaltes entspricht dem deutschen Leitbild parlamentarischer Außenpolitik in einer Art und Weise, die eine Reduzierung parlamentarischer Kontrollrechte im Hinblick auf eine weitere Integration multinationaler Streitkräfte, zum Beispiel in Form einer europäischen Armee, als unrealistisch erscheinen lässt. In diesem Zusammenhang ist es wenig überraschend, dass mit Ausnahme der Partei Die Linke eine vertiefte Integration europäischer Streitkräfte fraktionsübergreifend begrüßt wird und damit einhergehend die Aufwertung der Kontrollrechte anderer nationaler Parlamente sowie insbesondere jener des Europäischen Parlaments vehement gefordert wird. ${ }^{45}$ Darüber hinaus sehen die befragten Bundestagsabgeordneten eine hinreichende demokratische Legitimität der GSVP gegeben und erteilen einem demokratischen Defizit, zumindest in Deutschland, eine generelle Absage. Gleichzeitig sehen die Abgeordneten im deutschen Leitbild parlamentarischer Außenpolitik ein positives Beispiel für eine effiziente Parlamentskontrolle, dass als Modell für andere Länder dienen könnte, um die demokratische Legitimität der GSVP insgesamt zu stärken. Doch ist das normative Legitimitätsmodell des konstitutiven Parlamentsvorbehalts ohne Weiteres auf andere Länder übertragbar? Oder könnte ein zu starkes Maß an Parlamentsbeteiligung im Rahmen von internationalen Einsätzen in der Praxis auch schnell an Grenzen stoßen? Um diese Fragen qualifiziert beantworten zu können, wird im Folgenden die Rolle des britischen Parlaments in der GSVP näher untersucht.

43 Vgl. Hans Born / Heiner Hänggi, a.a.O. (Fn. 2), S. 8.

44 Sandra Dieterich / Hartwig Hummel / Stefan Marschall, Von der exekutiven Prärogative zum parlamentarischen Frieden? Funktionslogik und Funktionsprobleme der parlamentarischen Kontrolle militärischer Sicherheitspolitik am Fallbeispiel Deutschland (Paks Working Paper 6), Düsseldorf 2007, S. 20.

45 Vgl. Interviews im Deutschen Bundestag, a.a.O. (Fn. 6), S. 1 ff. 


\subsection{Großbritannien: Konstruktiver Partner der Exekutive im Rahmen der GSVP?}

Anders als der Bundestag hat das House of Commons keine so weitreichenden Kontrollmöglichkeiten in der GSVP. Die Entscheidung, britische Soldaten in Krisenregionen zu schicken, ist eine königliche Prärogative und unterliegt gewohnheitsrechtlich dem Ermessen des Premierministers. ${ }^{46}$ Gerichte haben keine Befugnisse, über eine derartige Entscheidung der Exekutive zu befinden, so dass die Regierung über ein hohes Maß an Flexibilität in Sicherheits- und Verteidigungsfragen verfügt. ${ }^{47}$ Vor diesem Hintergrund stellt die zunehmende Institutionalisierung einer europäischen Sicherheits- und Verteidigungspolitik eine besondere Herausforderung für das politische System Großbritanniens dar. Mit dem Beitritt zur Europäischen Gemeinschaft (EG) im Jahr 1973 wurde eine engere Zusammenarbeit der Mitgliedstaaten in der Außen- und Sicherheitspolitik grundsätzlich von der britischen Regierung begrüßt. Dies war aber nicht gleichbedeutend damit, eine verstärkte europäische Integration in diesem Bereich zu verfolgen. Vielmehr handelte es sich um einen pragmatischen Ansatz mit dem Ziel, unter Beibehaltung der nationalen Souveränität eine Führungsrolle in der EG auf diesem Feld zu übernehmen, um die britische Rolle im Machtgefüge einer bipolaren Welt zu untermauern. ${ }^{48}$

Die britische Unterstützung zum Projekt der GSVP zeigte sich nicht zuletzt in der Erklärung von St. Malo im Jahr 1998. Auf dem von Premierminister Tony Blair initiierten Treffen mit dem französischen Präsidenten Jacques Chirac befürwortete Großbritannien das Ziel der EU, die GASP um eine autonome Verteidigungskomponente zu ergänzen und diese mit angemessenen Planungs- und Entscheidungsverfahren zu versehen. Gleichzeitig verfolgt das Land bis heute vehement die Beibehaltung der intergouvernementalen Struktur der GSVP und lehnt eine weitere Integration der Streitkräfte auf europäischer Ebene konsequent ab. ${ }^{49}$ Stattdessen befürwortet das Vereinigte Königreich eine enge Beziehung mit der NATO und insbesondere mit den Vereinigten Staaten, um seine Fähigkeiten für eine weltweite Intervention aufrecht zu erhalten. ${ }^{50}$

Gerade vor diesem Hintergrund nimmt das demokratisch gewählte House of Commons, ebenso wie das nicht gewählte House of Lords, die Kontrolle der Sicherheits- und Verteidigungspolitik ernst. ${ }^{51}$ Als wichtiges Kontrollinstrument fungiert hierbei das European Scrutiny Committee (ESC), das alle EU-Dokumente und Entscheidungen erhält und überprüft. Das ESC äußert seine Meinung zur politischen und rechtlichen Bedeutung jedes einzelnen Dokuments und gibt es anschließend entweder frei, leitet es zur Behandlung in einen der drei mit EU-Angelegenheiten befassten Ausschüsse weiter oder empfiehlt eine Debatte im Plenum des Unterhauses. Auch wenn grundsätzlich alle Dokumente dem ESC vorgelegt

46 Vgl. Thomas Poole, The Royal Prerogative, in: International Journal of Constitutional Law, 8. Jg. (2010), H. 1, S. 146 - 155.

47 Vgl. Nicolai von Ondarza / Michael Klemm, Europeans in Afghanistan. Decision-making Processes in Britain, France and Germany (ECPR General Conference 2009), Potsdam 2009, S. 10.

48 Vgl. David Allen, British Parliamentary Participation in Foreign, Security and Defence Policy: Adapting to Europe within a Transformed World, in: Jürgen Mittag, The Parliamentary Dimension of CFSP/ESDP. Options for the European Convention, Brüssel / Köln 2009, S. 147 - 169, S. 147.

49 Vgl. Julian Böcker, a.a.O. (Fn. 1), S. 186 ff.

50 Vgl. David Allen, a.a.O. (Fn. 48), S. 148.

51 Vgl. Interviews im britischen Unterhaus (House of Commons), a.a.O. (Fn. 6), S. 67 ff. Ergänzend hierzu vgl. Interviews im britischen Oberhaus (House of Lords), ebenda, S. 98 ff. 
werden, so fallen diejenigen, die sich auf die Außen- und Sicherheitspolitik beziehen, oftmals durch dieses Schema. ${ }^{52}$ Einer der Gründe dafür liegt darin, dass Entscheidungen über GSVP-Einsätze weder legislativen Charakter haben noch rechtlich bindend sind. Folglich legt die britische Regierung dem ESC Schlussfolgerungen des Rates erst im Nachhinein vor und umgeht somit in der Regel eine parlamentarische ex ante-Kontrolle. Oftmals werden Entscheidungen im Vorfeld von Ratssitzungen auch als vertraulich kategorisiert und können deshalb nicht veröffentlicht werden. ${ }^{53}$

Zwar führt dieses stark institutionalisierte System dazu, dass das Unterhaus weit reichende Kontrollmöglichkeiten für jegliche EU-Gesetzgebung hat; der Einfluss des ESC auf die meisten Initiativen im Bereich der Sicherheits- und Verteidigungspolitik ist aber deutlich begrenzt. Eine Vielzahl der Dokumente mit sicherheitspolitischer Relevanz wird vielmehr gemeinsam mit nationalen Entscheidungen im Verteidigungsausschuss und im Ausschuss für Auswärtige Angelegenheiten diskutiert. ${ }^{54}$ Beide Ausschüsse debattieren grundsätzlich Außen- und Sicherheitspolitik und veröffentlichen Berichte, die sowohl auf schriftlichen als auch auf mündlichen Auskünften von Ministern, Verwaltungsbeamten und Experten beruhen. Während der Verteidigungsausschuss in erster Linie das Handeln des Verteidigungsministeriums untersucht, beschäftigt sich der Ausschuss für Auswärtige Angelegenheiten mit den Aktivitäten des Foreign and Commonwealth Office (FCO), das primär für die GSVP zuständig ist. ${ }^{55}$ Dies führt dazu, dass die GSVP im Verteidigungsausschuss eine untergeordnete Rolle spielt, da es hier stärker um die nationale Verteidigungspolitik geht. Auch wenn der Verteidigungsausschuss das allgemeine britische Militärbudget kontrolliert und darüber entscheidet, ist er nicht befugt, Haushaltsposten für einzelne GSVP-Missionen zu genehmigen. ${ }^{56}$

Die wesentlichen Einflussmöglichkeiten für das Unterhaus beschränken sich somit auf die ex post-Kontrolle der Außen- und Sicherheitspolitik. Diese findet durch eine ausführliche Berichterstattung statt, nachdem die Soldaten bereits in eine Krisenregion entsandt worden sind. ${ }^{57}$ Die dominante Rolle der Exekutive auf diesem Politikfeld gerät jedoch zunehmend in die Kritik. Auslöser stellt die britische Teilnahme am Irakkrieg im Jahr 2003 dar. Bereits vor Amtsantritt der Labour-Regierung 1997 hatten einige spätere Mitglieder des Kabinetts von Tony Blair die königliche Prärogative als nicht mehr zeitgemäß kritisiert. ${ }^{58}$ Im Zuge der damaligen Diskussion ließ Blair am 18. März 2003 erstmals seit dem Koreakrieg 1950 das House of Commons über den Einsatz von Soldaten abstimmen. Das Unterhaus votierte mit einer großen Mehrheit von 412 zu 149 Stimmen dafür, wobei Blair die Abstimmung jedoch informell mit einer Art Vertrauensfrage verknüpft hatte. Zudem muss die von der Regierung seinerzeit betriebene Informationspraxis als unzureichend bezeichnet werden. ${ }^{59}$

52 Vgl. Flavia Zanon, EU Foreign Policy Cooperation: A Challenge for National Parliaments?, in: European Foreign Affairs Review, 15. Jg. (2010), H. 1, S. 19 - 38, S. 21.

53 Vgl. Hans Born / Alex Dowling / Teodora Fuior / Suzana Gavrilescu, a.a.O. (Fn. 27), S. 47.

54 Vgl. Flavia Zanon, a.a.O. (Fn. 52), S. 21 f.

55 Vgl. David Allen, a.a.O. (Fn. 48), S. 154.

56 Vgl. Sandra Dieterich / Hartwig Hummel / Stefan Marschall, a.a.O. (Fn. 1), S. 16.

57 Vgl. Nicolai von Ondarza / Michael Klemm, a.a.O. (Fn. 47), S. 10.

58 Vgl. Keth Syrett, Prerogative Powers: New Labour's Forgotten Constitutional Reform?, in: Denning Law Journal, 13. Jg. (1998), H. 1, S. 111 - 129, S. 117.

59 Vgl. Alan Doig, 45 Minutes of Infamy? Hutton, Blair and the Invasion of Iraq, in: Parliamentary Affairs, 58. Jg. (2005), H. 1, S. 109 - 123; Steve Kettell, The Curious Incident of the Dog that 
Mit Premierminister Gordon Brown gewann die Diskussion weiter an Dynamik. Dessen Regierung präsentierte nur wenige Wochen nach Amtsantritt im Jahr 2007 ein ambitioniertes Reformvorhaben unter dem Titel "The Governance of Britain "60. Das so genannte Green Paper sah vor, die Befugnisse der Regierung zur Entsendung von Truppen in bewaffnete Konflikte zu beschneiden und an die Zustimmung des Parlaments zu binden. Im 21. Jahrhundert sei die königliche Prärogative in diesem sensiblen Bereich schlichtweg „out of date“61. Im Oktober 2007 folgte ein detailliertes Konsultationspapier mit dem Titel „The Governance of Britain. War Powers and Treaties: Limiting Executive Powers“ ${ }^{\text {62 }}$. Die Regierung präsentierte darin vier verschiedene Modelle zur Formalisierung der parlamentarischen Beteiligungsrechte im Falle eines Streitkräfteeinsatzes. Jedes Modell sah dabei explizit vor, dass eine Entsendung von Streitkräften durch die Regierung vorab einer Autorisierung durch das House of Commons bedarf. ${ }^{63}$ Das daran anschließende Konsultationsverfahren mündete schließlich in der Vorlage eines umfangreichen Entwurfs für eine Resolution des Parlaments. ${ }^{64}$ Zur Entsendung von Streitkräften ins Ausland sollte demnach der Premierminister einen Bericht an das House of Commons richten, der über die Ziele, den Ort und entsprechende Rechtsfragen des Einsatzes Auskunft geben sollte. Vor der Entscheidung über den Bericht sollte das House of Lords angehört werden.

Die auf den ersten Blick recht rigorose Regelung eines parlamentarischen Vorbehalts wäre jedoch durch verschiedene Ausnahmen relativiert worden. Liegt beispielsweise aus Sicht des Premierministers ein besonderer Notfall vor, sieht dieser die Effektivität des Einsatzes in Frage gestellt oder die Sicherheit der Operation gefährdet, sollte die Notwendigkeit einer Autorisierung durch das House of Commons entfallen. Nach der Einsatzentscheidung sollte das Parlament informiert werden, es sei denn, Sicherheitsbedenken des Premierministers hätten dagegen gesprochen. Eine Zustimmung wäre ebenfalls nicht erforderlich, wenn das Unterhaus zum betreffenden Zeitpunkt aufgelöst wäre. Die Entsendung von Spezialkräften sollte grundsätzlich von jeder Form der Zustimmung oder Information ausgenommen werden. Die angestrebte Reform fand unter der Administration von Gordon Brown jedoch keinen Abschluss. ${ }^{65}$ So wurde in dem Constitutional Reform and Governance Act 2010 die Frage der Parlamentsbeteiligung bei Auslandseinsätzen explizit ausgeklammert. Der seit 2010 amtierende konservative Premierminister David Cameron scheint aber ebenfalls daran interessiert zu sein, durch eine Resolution eine Konvention zu etablieren. ${ }^{66}$ So ließ er das House of Commons am 21. März 2011 erfolgreich über einen militärischen Einsatz in Libyen abstimmen; die Abstimmung erfolgte jedoch erst zwei Tage nach der Entsendung

Didn't Bark in the Night-Time: Structure and Agency in Britain's War with Iraq, in: Politics \& Policy, 37. Jg. (2009), H. 2, S. $415-439$.

60 Lord Chancellor / Secretary of State for Justice, The Governance of Britain (CM 7170), London 2007.

61 Ebenda, S. 15.

62 Lord Chancellor / Secretary of State for Justice, The Governance of Britain. War Powers and Treaties: Limiting Executive Powers (CM 7239), London 2007.

63 Vgl. ebenda, S. 39.

64 Vgl. Lord Chancellor / Secretary of State for Justice, The Governance of Britain - Constitutional Renewal (CM 7342-I), London 2008, S. 53 ff.

65 Vgl. Matthew Flinders, Bagehot Smiling: Gordon Brown's 'New Constitution' and the Revolution that Did Not Happen, in: The Political Quarterly, 81. Jg. (2010), H. 1, S. 57 - 73.

66 Vgl. Johannes Hinz, Die Rolle der Parlamente beim Auslandseinsatz deutscher und britischer Streitkräfte, in: ZParl, 42. Jg. (2011), H. 2, S. 286 - 306, S. 294. 
britischer Truppen. Zwar entschied das Unterhaus mit 557 zu 13 Stimmen deutlich zugunsten Camerons; vereinzelt äußerten Abgeordnete aber Kritik hinsichtlich des Vorgehens der Regierung. ${ }^{67}$

Die britische Regierung verfügt somit bis heute über ein hohes Maß an Flexibilität in Sicherheits- und Verteidigungsfragen. Auch seitens des Parlaments wird an der intergouvernementalen Struktur der GSVP aus zwei wesentlichen Gründen festgehalten. Zum einen behalten die Abgeordneten, die die Exekutive der Regierung in der Außen- und Sicherheitspolitik mit einigen Ausnahmen grundsätzlich anerkennen, so ihre Vorreiterrolle bei der Regierungskontrolle. ${ }^{68}$ Im Gegensatz zu ihren deutschen Kollegen erteilen britische Abgeordnete einer Aufwertung des Europäischen Parlaments im Entscheidungsprozess der GSVP eine klare Absage. Durch eine Ausweitung der Kompetenzen des EP befürchten viele vor allem eine Einschränkung der Entscheidungsmöglichkeiten der britischen Regierung, die als demokratisch gewähltes Organ primär die nationalen Positionen zu vertreten habe. Zum anderen sieht Großbritannien seine sicherheitspolitischen Interessen nicht in der EU, sondern in der NATO am besten aufgehoben. Anders als Deutschland gebe sich das Land nicht mit Aktivitäten in Post-Konflikt-Operationen oder im Grenzschutz zufrieden. Vielmehr gehe es um die Fähigkeit, militärische Macht zu projizieren und dabei so autonom wie möglich zu bleiben. ${ }^{69}$ Auch wenn das Vereinigte Königreich neuerdings eine enge bilaterale Kooperation mit Frankreich betreibt und die übrigen europäischen Partner nachdrücklich dazu auffordert, ihre Fähigkeiten im Bereich des Krisenmanagements zu verbessern, so wird der GSVP lediglich ein komplementärer Charakter zugebilligt. Folglich rückt aus britischer Sicht der Stellenwert einer parlamentarischen Beteiligung innerhalb des Entscheidungsprozesses der GSVP in den Hintergrund und hat in den Augen der befragten Abgeordneten keine wesentlichen Auswirkungen auf die demokratische Legitimität dieser Politik. Allerdings mehren sich mittlerweile die Stimmen, die die königliche Prärogative für nicht mehr zeitgemäß halten und daher eine stärkere formale Beteiligung des britischen Unterhauses verlangen.

\subsection{Europäisches Parlament: Garant einer demokratisch legitimierten GSVP?}

Das Europäische Parlament hat keine rechtlich verbindlichen Einflussmöglichkeiten auf militärische GSVP-Missionen. Seine durch den Vertrag von Lissabon verstärkten haushaltspolitischen Kompetenzen finden nur bei zivilen GSVP-Missionen Anwendung, da militärische Operationen über den ATHENA-Mechanismus ${ }^{70}$ von den Mitgliedstaaten finanziert

67 Vgl. House of Commons, Operations in Libya. Ninth Report of Session 2010-12, London 2012, S. 15.

68 Vgl. Interviews im britischen Unterhaus (House of Commons), a.a.O. (Fn. 6), S. 67 ff. Ergänzend hierzu vgl. Interviews im britischen Oberhaus (House of Lords), ebenda, S. $98 \mathrm{ff}$.

69 Vgl. Ronja Kempin / Jocelyn Mawdsley / Stefan Steinicke, Entente Cordiale. Eine erste Bilanz französisch-britischer Zusammenarbeit in der Sicherheits- und Verteidigungspolitik (DGAPanalyse 10), Berlin 2012, S. $11 \mathrm{f}$.

70 ATHENA wurde 2004 als Mechanismus zur Verwaltung der Finanzierung gemeinsamer Kosten der Operationen der EU mit militärischen oder verteidigungspolitischen Bezügen eingerichtet. Er wird unter Aufsicht eines Sonderausschusses verwaltet, der sich aus Vertretern der beitragenden Mitgliedstaaten zusammensetzt (Beschluss 2011/871/GASP des Rates vom 19. Dezember 2011). 
werden und damit lediglich der Kontrolle der nationalen Parlamente unterliegen. Dennoch ist die Rolle des EP in der GSVP nicht zu unterschätzen. Aufgrund seines Standortvorteils und seiner daraus resultierenden physischen Nähe zu den relevanten EU-Institutionen sowie des im Jahre 2004 speziell für die parlamentarische Kontrolle der GSVP eingerichteten Unterausschusses für Sicherheit und Verteidigung (SEDE) verfügen die Mitglieder des Europäischen Parlaments (MdEPs) über weit bessere Informationen als viele ihrer Kollegen in den nationalen Parlamenten. Obwohl der SEDE bislang nur ein Unterausschuss mit beschränkten parlamentarischen Möglichkeiten ist, so ist durch seine Spezialisierung eine konstante Befassung mit der GSVP auf europäischer Ebene gegeben. Diese findet durch eine Vielzahl an Anhörungen und Debatten im Ausschuss für Auswärtige Angelegenheiten (AFET) und im SEDE, durch Truppenbesuche der MdEPs in den Einsatzgebieten, durch verschiedene interinstitutionelle Vereinbarungen, die den Zugang zu vertraulichen Dokumenten für einen ausgewählten Kreis an MdEPs ermöglichen, sowie durch formelle und informelle Netzwerke statt. ${ }^{71}$

Die Abgeordneten haben damit nicht nur den Vorteil, Informationen auf kürzestem Wege aus dem Rat und der Europäischen Kommission zu erhalten. Aufgrund der Tatsache, dass dem EP Vertreter aus allen EU-Mitgliedstaaten angehören, kommt es zu einem ständigen Dialog unter Kollegen, die teilweise ganz unterschiedliche Auffassungen zur Ausgestaltung der GSVP und insbesondere zur Rolle der demokratisch gewählten Parlamente aufweisen. Als Spiegelbild von 27 Demokratieverständnissen und Legitimitätsvorstellungen wundert es daher nicht, dass die Resolutionen des EP zur GSVP einen Kompromiss entlang existierender parlamentarischer Kontrollregime formulieren. So wird beispielsweise in der Entschließung vom 10. März 2010 weder die intergouvernementale Struktur der GSVP infrage gestellt noch ein mögliches Demokratiedefizit bemängelt. ${ }^{72}$ Allerdings fordert das EP eine größere und transparentere Informationspolitik seitens der nationalen Regierungen und damit eine Intensivierung der bereits verstärkten Zusammenarbeit zwischen Rat und EP. Das Europäische Parlament bestätigt ferner seine Monopolstellung in Bezug auf die supranationale verfahrensorientierte Legitimität und befürwortet die Entwicklung einer engen und strukturierten Zusammenarbeit mit den nationalen Parlamenten.

Eine Aufwertung der parlamentarischen Kontrollrechte in Form eines konstitutiven Parlamentsvorbehalts wird jedoch nicht gefordert. ${ }^{73}$ Die primäre Verantwortung für die Bereitstellung der demokratischen Legitimität obliege weiterhin den nationalen Parlamenten. Erst mit der Entwicklung einer europäischen Armee würde es notwendig, GSVP-Missionen aus dem EU-Haushalt zu finanzieren und vom EP direkt kontrollieren zu lassen. Auch wenn manche Abgeordnete das langfristige Ziel einer Parlamentarisierung der GSVP begrüßen, war der Großteil der befragten Abgeordneten der Auffassung, dass das Verhältnis zwischen Rat und Europäischem Parlament als ein stetiger Prozess der Annäherung charakterisiert werden müsse. Dieser Prozess könne aufgrund von unterschiedlichen Legitimitätsvorstellungen nur durch Änderungen der nationalen parlamentarischen Traditionen zu einer rechtlichen Anpassung auf europäischer Ebene führen. Sofern die nationalstaatlichen Entschei-

71 Vgl. Nicolai von Ondarza / Michael Klemm, a.a.O. (Fn. 47), S. 2.

72 Vgl. Europäisches Parlament, Entschließung des Europäischen Parlaments zu der Umsetzung der Europäischen Sicherheitsstrategie und die Gemeinsame Sicherheits- und Verteidigungspolitik (2009/2198(INI)), Brüssel 2010, S. 24.

73 Vgl. Interviews im Europäischen Parlament, a.a.O. (Fn. 6), S. 145 ff. 
dungsabläufe eingehalten werden, könne man somit von einer ausreichenden demokratischen Legitimität der GSVP ausgehen. Auch wenn die Kontrolle aus Sicht der MdEPs primär den nationalen Parlamenten unterliegt, kann man zweifellos konstatieren, dass das Europäische Parlament eine wichtige, zusätzliche Ebene demokratischer Legitimität bereitstellt, die durch eine Aufwertung des SEDE zu einem vollwertigen Ausschuss noch gesteigert werden könnte. Ferner sind die nationalen Parlamente und das EP gut beraten, nach der Auflösung der Westeuropäischen Union (WEU), eine endgültige Entscheidung über ihre zukünftige $\mathrm{Zu}$ sammenarbeit baldmöglichst zu treffen, um den gegenseitigen Informationsaustausch sowie die Koordinierung und Vernetzung miteinander zu verbessern.

\section{Ausblick: Ein effektives Mehrebenenmodell parlamentarischer Kontrolle}

Deutschland und Großbritannien weisen zwei nationale Varianten der parlamentarischen Kontrolle im Bereich der europäischen Sicherheits- und Verteidigungspolitik auf, deren Unterschiede auf die historische Entwicklung und damit die verschiedenen Demokratiemodelle und Legitimitätsverständnisse zurückzuführen sind. Ihre vergleichende Darstellung unter Einbeziehung der formalen Kompetenzen des Europäischen Parlaments bei der Kontrolle der GSVP und der informellen Mitwirkungsmöglichkeiten hat deutlich gezeigt, dass Sicherheits- und Verteidigungspolitik in der EU grundsätzlich keine isolierten Handlungsbereiche der nationalstaatlichen Exekutiven mehr sind. Schon formal verfügen alle drei ausgewählten Parlamente über mehr Rechte und Einflusschancen, als vielfach in der Diskussion wahrgenommen wird. Die These des doppelten Demokratiedefizits muss daher kritisch hinterfragt werden. Gleichwohl ergeben die unterschiedlichen Formen der parlamentarischen Kontrolle im Bereich der GSVP kein einheitliches Bild. In Deutschland haben insbesondere das Urteil des Bundesverfassungsgerichts von 1994 und das daraus resultierende Parlamentsbeteiligungsgesetz die Mitwirkungsmöglichkeiten des Bundestages erheblich erweitert und die grundsätzliche parlamentarische Auseinandersetzung mit sicherheits- und verteidigungspolitischen Fragen befördert. Die verfassungsrechtliche Notwendigkeit einer konstitutiven Zustimmung des Bundestages vor jedem Einsatz nationaler Streitkräfte wurde somit zum Leitmotiv des deutschen Legitimitätsverständnisses in der Sicherheits- und Verteidigungspolitik.

Das britische Verständnis parlamentarischer Kontrolle unterscheidet sich grundlegend vom deutschen Modell. In Großbritannien sind es allein der Premierminister und seine Regierung, die in der Tradition der königlichen Prärogative über den Einsatz von Soldaten entscheiden. Das Unterhaus spielt hier nur eine untergeordnete Rolle. Gleichzeitig wird der Regierung von Seiten des Parlaments ein hohes Maß an Flexibilität in Sicherheits- und Verteidigungsfragen zugebilligt. Erst nach der Teilnahme britischer Streitkräfte am Irakkrieg im Jahr 2003 hat sich das House of Commons stärker in die Formulierung der nationalen Sicherheits- und Verteidigungspolitik eingebracht. Anders als in Deutschland mündete dieser Prozess jedoch bislang noch nicht in einer Institutionalisierung der ex ante-Kontrolle in Form eines parlamentarischen Vorbehalts. Mit Blick auf die europäische Ebene stellt die intergouvernementale Ausgestaltung der GSVP für britische Abgeordnete sogar einen Garant demokratischer Legitimität dar. Eine weitere Integration in diesen Bereich und eine damit möglicherweise verbundene Ausdehnung von Kompetenzen des Europäischen Parlaments lehnen sie in Übereinstimmung mit ihrer Exekutive sogar vehement ab. Die 
Unterstellung eines doppelten Demokratiedefizits und die Forderung nach einer verstärkten Parlamentarisierung der GSVP widersprechen somit inhärent dem britischen Legitimitätsverständnis.

Trotz der primärrechtlich verankerten eher marginalen Rolle des Europäischen Parlaments im intergouvernementalen Bereich der GSVP steht dieses dem Rat keineswegs mittellos gegenüber. Im Vergleich zu den nationalen Parlamenten hat das EP einen enormen Standortvorteil und ist, unter anderem durch regelmäßige Debatten mit verantwortlichen nationalen Ministern oder der Hohen Vertreterin der EU für Außen- und Sicherheitspolitik, prinzipiell besser über die Entwicklungen im Bereich der GSVP informiert. Die nationalen Parlamente haben in der Regel keinen direkten Zugang zu den europäischen Institutionen und sind überwiegend auf den Informationsfluss ihrer Regierungen angewiesen. Im Sinne einer effektiven parlamentarischen Kontrolle reicht es jedoch nicht aus, lediglich über die Gegenstände europäischer Verhandlungen und Entscheidungen informiert zu sein. Um zwischen möglichen Verhandlungsspielräumen und Entscheidungsoptionen abwägen zu können, haben die nationalen Parlamente daher verschiedene Wege der interorganisatorischen Vernetzung eingeschlagen. Dies betrifft sowohl die Zusammenarbeit zwischen den einzelnen Parlamenten der Mitgliedstaaten als auch den gegenseitigen Informations- und Erfahrungsaustausch zwischen ihnen und dem EP.

Neben einem kontinuierlichen informellen Austausch über die jeweiligen nationalen Parteiorganisationen oder Fraktionen findet interparlamentarische Zusammenarbeit auch in institutionalisierter Form statt. Gerade im Rahmen der durch den Vertrag von Lissabon neu geschaffenen Subsidiaritätskontrolle bemühen sich sowohl die Konferenz der Europaausschüsse (COSAC) als auch das EP und die Kommission um Verfahrensvorschläge für eine verstärkte interparlamentarische Koordination, die auch den Bereich der GSVP einschließen soll. Der Vertrag von Lissabon sieht mit dem Protokoll über die Rolle der nationalen Parlamente in der EU explizit die Möglichkeit vor, Beschlüsse zur interparlamentarischen Zusammenarbeit auch im Bereich der Sicherheits- und Verteidigungspolitik zu fassen. Im Zentrum der Diskussion stand lange Zeit eine Aufwertung der Parlamentarischen Versammlung der WEU. Da die EU seit Lissabon erstmals auch über eine militärische Beistandsklausel für ihre Mitgliedstaaten verfügt, wurde die WEU als kollektiver Beistandspakt jedoch vollends obsolet und daher im Juni 2011 aufgelöst. Um diesem Schicksal zu entgehen, hatte die Parlamentarische Versammlung der Organisation noch im Jahr 2008 ihren Namen in Europäische Versammlung für Sicherheit und Verteidigung (ESDA) geändert und damit ihren Anspruch artikuliert, auch als interparlamentarisches Gremium der GSVP zu fungieren. Ungeachtet dieser Initiative fand die letzte ESDA-Vollversammlung schließlich ebenfalls im Juni 2011 in Paris statt. Zwar wurde die Organisation als Europäische Sicherheits- und Verteidigungsvereinigung (ESDA) im Oktober 2011 neu gegründet, jedoch versteht sich die ESDA nun stärker als Diskussionsforum und weniger als eine tatsächliche parlamentarische Versammlung für den Bereich der Sicherheits- und Verteidigungspolitik.

Wie die durch den Vertrag von Lissabon und die Auflösung der WEU entfachte Reformdebatte am Ende auch ausgehen mag: Jede Initiative zur Stärkung der parlamentarischen Kontrolle der GSVP wird den individuellen Legitimitätskonzepten von 27 EU-Mitgliedstaaten Rechnung tragen müssen. Diese sind, das haben das deutsche und britische Beispiel gezeigt, höchst unterschiedlich und schließen sich zum Teil aus. Ein einzelnes, allgemeingültiges Modell der parlamentarischen Kontrolle europäischer Sicherheits- und Verteidigungspolitik existiert zum jetzigen Zeitpunkt nicht. Die Diskussion über das vermeintliche 
doppelte Demokratiedefizit der GSVP basiert somit auf einem kontrafaktischen Legitimitätsmodell parlamentarischer Kontrolle, das die empirische Realität innerhalb der EU nicht hinreichend reflektiert.

Bedingt durch den komplementären Charakter der nationalen Parlamente und des Europäischen Parlaments ist die europäische Sicherheits- und Verteidigungspolitik von einer Vielzahl unterschiedlicher Kontrollregime gekennzeichnet, deren individuellen Praktiken zu einem effektiven Mehrebenenmodell parlamentarischer Kontrolle der GSVP zusammenzuführen sind. Es bleibt zu hoffen, dass die letztlich im Rahmen des Protokolls über die Rolle nationaler Parlamente gefundenen institutionellen Arrangements zur Förderung der interparlamentarischen Zusammenarbeit die GSVP tatsächlich transparenter und greifbarer für die europäischen Bürger machen und damit positiv zur demokratischen Legitimität beitragen. Solange aber in jedem Mitgliedsstaat der EU die konstitutionellen und demokratischen Entscheidungsabläufe in Bezug auf die GSVP eingehalten werden - sei es die konstitutive Zustimmung oder lediglich die ex post-Konsultation eines Parlaments - solange muss unter Berücksichtigung der unterschiedlichen nationalen Demokratieverständnisse und den damit verbundenen Legitimitätsvorstellungen der Vorwurf eines doppelten Demokratiedefizits zurückgewiesen werden.

\section{Jahre nach dem ersten Misstrauensantrag im Deutschen Bundestag: Probleme einer Minderheitsregierung am Beispiel der ersten Regierung Willy Brandts}

Jürgen Plöhn

2012 jährte sich zum 40. Mal die Abstimmung über den ersten Misstrauensantrag der Opposition im Deutschen Bundestag und die erste Vertrauensfrage eines Bundeskanzlers. Am 18. Dezember 2013 wird des 100. Geburtstages Willy Brandts zu gedenken sein. Für Politologen rechtfertigen Jahrestage jedoch nur dann die Erinnerung an zurückliegende Ereignisse, wenn diesen eine fortgesetzte politische Relevanz zukommt. Nach angelsächsischer Tradition gilt das besonders für Präzedenzfälle. Die dramatischen parlamentarischen Ereignisse des Jahres 1972, die in diese Kategorie fallen, sind zwar bereits zeitnah und nachfolgend wiederholt dargestellt worden, die Einordnung der Regierung Brandts als Minderheitsregierung verspricht indes zusätzliche Erkenntnisse.

\section{Zur Abgrenzung: Was ist eine "Minderheitsregierung“?}

Von einer Minderheitsregierung unter Willy Brandt ist in der zeitgeschichtlichen und politikwissenschaftlichen Literatur kaum etwas bekannt ${ }^{1}$. Die parlamentarische Lage im Som-

1 Ludger Helms, Politische Opposition, Opladen 2002, S. 52, nennt die Ereignisse von November/ Dezember 1962 (Konrad Adenauer), Oktober/November 1966 (Ludwig Erhard) und September 1982 (Helmut Schmidt) als die drei einzigen bis 2002 in Deutschland auf Bundesebene aufgetretenen Minderheitsregierungen. 